\title{
THEORETICAL STUDY ON LINKAGE UNIT OF WIDE SPAN TRACTOR
}

\author{
Volodymyr Bulgakov, Viktor Melnik, Volodymyr Kuvachov \& Jüri Olt
}
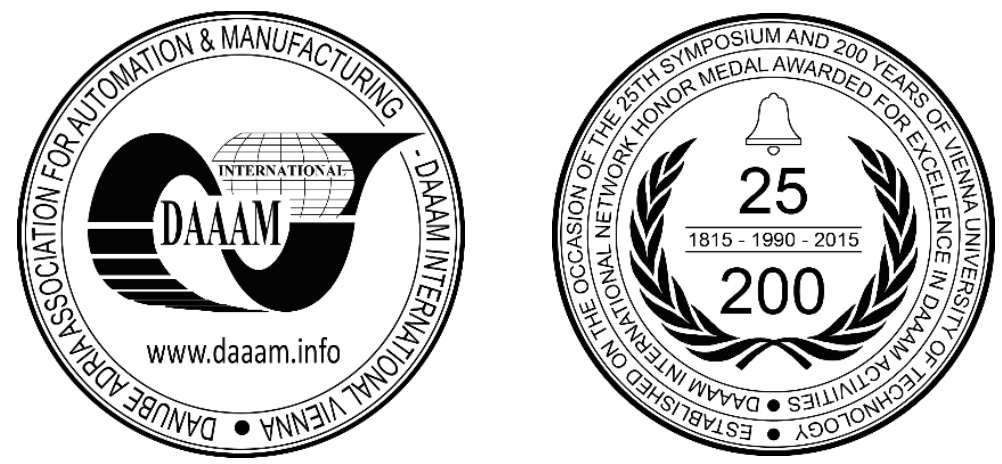

This Publication has to be referred as: Bulgakov, V[olodymyr]; Melnik, V[iktor]; Kuvachov, V[olodymyr] \& Olt, J[ueri] (2018). Theoretical Study on Linkage Unit of Wide Span Tractor, Proceedings of the 29th DAAAM International Symposium, pp.0180-0189, B. Katalinic (Ed.), Published by DAAAM International, ISBN 978-3-902734-20-4, ISSN 1726-9679, Vienna, Austria

DOI: $10.2507 / 29$ th.daaam.proceedings.026

\begin{abstract}
In order to ensure the stable motion of the wide span tractor, the design of its linkage has to provide for its independent turning on the horizontal plane. That implies the high priority of the issue of substantiating the parameters of the wide span tractor linkage unit. The aim of the study was to improve the efficiency of operation and application of wide span tractors through the substantiation of the parameters of their linkage units. The study uses as its basis the fundamental principles of the theoretical mechanics, the theory of tractor, the statistical dynamics and the theory of automatic control of linear dynamic systems responding to statistically random controlling and perturbing input actions. Following the results of the undertaken investigations, it has been established that the most desirable setup of the wide span tractor's linkage unit in terms of the tractor's response to the controlling action (angular displacement of steering wheels) and perturbing action (angular displacement of linkage unit on the horizontal plane) is when the instantaneous centre of turn situated at the point of convergence of the centrelines of the lower draught links coincides with the tractor's centre of mass.
\end{abstract}

Keywords: wide span tractor; linkage unit; stability and controllability; angular mobility

\section{Introduction}

Recently, the dedicated wide-track agricultural machines (which most frequently means wide span tractors) have been acquiring ever greater worldwide popularity [1], [2] and [9]. The latter units enable implementing the controlled traffic, gantry and precision farming technologies, while their development is aligned with the vector of scientific and technological progress, in particular, in terms of the automation and robotization of the technology-intensive farming.

In the view of their rather high versatility, wide span tractors can (and surely have to) be ganged up with trailing, semimounted and fully mounted agricultural implements and tools [3], [4], [5] and [6]. At the same time, the design layout of the wide span tractor suggests that the process unit is preferably connected to it (with the use of a linkage unit or without it) sufficiently close to the tractor's centre of mass [7], [8] and [9]. Under such circumstances, the problem is to avoid the negative effect of the wide span tractor's course deviations on the stability of motion of the attached machines and implements. 
For that purpose, the design of the linkage unit shall provide for their independent (to a certain extent) turning on the horizontal plane. Whereby, the issue of substantiating the parameters of the linkage unit of the wide span tractor in terms of the stable motion of its process unit on the horizontal plane is of great concern. This study investigates into the details of hitching wide span tractors with agricultural machines and implements. It is well-known that the three-point layout of the rear linkage gear in the conventional tractor can provide the sufficient mutual angular manoeuvrability of its frame and process unit. I.e. the turning of the tractor's frame resulting from the inputs to its operating controls will not interfere with the stable motion of the agricultural implement on the horizontal plane. That requires the lower draught links of the tractor's rear linkage unit converge at a convergence angle of at least $0.38 \mathrm{rad}$ (22 deg) [4]. In that case, the instantaneous centre of rotation of the tractor's linkage unit on the horizontal plane is close to its centre of mass. Due to the fact that in conventional tractors the distance from the line that passes through the points of attachment of the linkage unit's lower draught links to the centre of mass is considerably greater, than in case of wide span tractors, the lower draught link convergence angle is sufficiently small. Taking into account the possibility of unification of the linkage units used with wide span tractors and with conventional tractors, the parameters of which are regulated by the ISO 730:2009 international standard, it is necessary to establish the correspondence of sizes and requirements for the 3-point linkages intended for the attachment of agricultural implements and tools. The mentioned standard ISO 730:2009 defines various categories of linkage units operated with different agricultural tractors.

Alongside with that, recently certain trends have taken shape in the improvement of the design of 3-point linkage units for tractors [10], [11], [12], [13], [14], [15], [16] and [17]. However, the problem of their angular mobility is not given sufficient consideration, especially with regard to wide span tractors, which feature atypical design layout, principles of operation and operating conditions. The aim of the study was to improve the efficiency of operation and application of wide span tractors by substantiating the selection of the parameters of their linkage units.

\section{Materials and Methods}

The basis for the study is provided by the fundamentals of the theoretical mechanics, the theory of tractor, the statistical dynamics and the theory of the automatic control of linear dynamic systems responding to statistically random controlling and perturbing input actions. The analysis of the effect that the layout and parameters of the wide span tractor under investigation has on its controllability and dynamical stability is based on the amplitude and phase frequency response characteristics.

In the mathematical modelling of the motion of the wide span tractor on the horizontal plane, the dynamic model of its operation was made in the form of its response to the input controlling and perturbing actions. A dedicated agricultural wide span vehicle of the established design was taken as the physical object of study [4]. When the wide span tractor and its process unit perform two-dimensional motion on the horizontal plane, they are under the effect of a number of respective forces. In order to provide for the mathematical modelling of the wide span tractor's two-dimensional motion, its equivalent schematic model is to be constructed. (Fig. 1).

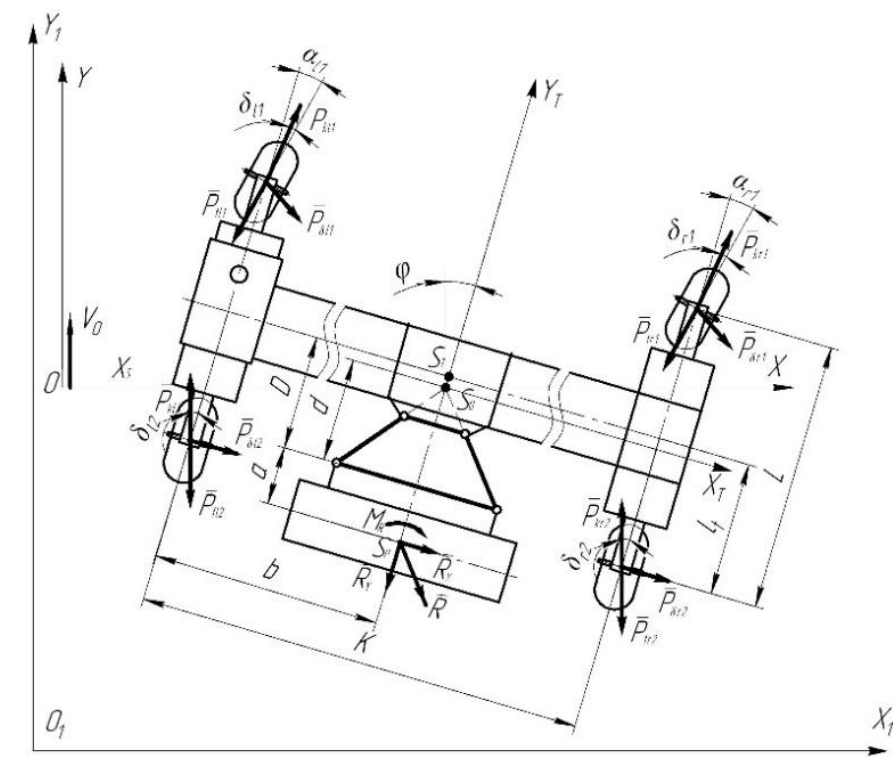

Fig. 1. Plan of forces acting on wide span tractor in horizontal plane.

The construction of the equivalent schematic model of the wide span tractor in two-dimensional motion (Fig. 1) is based on the following assumptions. On its working run, the tractor performs uniform translation at a velocity of $V_{0}$ with reference to the fixed horizontal plane $X_{1} O_{1} Y_{1}$. As the tractor and its process unit perform the work, they deviate, under the effect of random perturbations, from their original positions and acquire additional velocities. 
When that takes place, the tractor and the process unit perform relative motion in the XOY plane. At the same time, the $X_{T} S_{0} Y_{T}$ plane connected to the instantaneous centre of turn of the wide span tractor's linkage unit, which is situated at the point of convergence of the centrelines of the unit's lower draught links, turns relative to the $X O Y$ plane about the vertical axis that passes through the point $S_{0}$. The amount of the turn is represented by the angle $\varphi$ formed by the tractor's longitudinal line of symmetry $S_{0} Y_{T}$ and the axis $O Y$. During the relative motion, its centre of mass conventionally placed at the point $S_{0}$ performs movements on the $O X$ axis, which are represented by changes of the abscissa $X_{S}=O S_{0}$. Hence, the wide span tractor under investigation with a process unit attached to it has two degrees of freedom relative to the $X O Y$ plane represented by the two generalized coordinates: angle $\varphi$ and abscissa $X_{S}$.

\section{Results}

The differential equations of the wide span tractor's motion with reference to the $X O Y$ plane (Fig. 1) can be generated with the use of the original Lagrange equations of the second kind in the following form:

$$
\frac{d}{d t}\left(\frac{\partial T}{\partial \dot{q}_{i}}\right)-\frac{\partial T}{\partial q_{i}}=Q_{i} \quad(i=1,2)
$$

where $T$ - kinetic energy of mechanical system under consideration;

$q_{i}$ - generalized coordinate;

$i$ - number of generalized coordinates;

$Q_{i}-$ generalized force that corresponds to generalized coordinate $q_{i}$.

It should be noted that even in its linear interpretation the mathematical model of the wide span tractor's motion is represented by a system of rather complex differential equations. Therefore, in order to simplify their generation, the following assumptions are made: the tractor has neither heel nor trim; the wide span tractor is regarded as a physical solid body with the longitudinal plane of symmetry passing through its centre of mass; the variation of the tractive resistance of the agricultural implements has no effect on the translational velocity, whereby, it is assumed constant; the lateral interaction between the tractor's tyres and the surface being deformed (soil) is considered within the framework of the "lateral drift" hypothesis; the gyroscopic and stabilizing moments generated by the tyres and the moments of resistance to their torsion relative to the vertical axis are ignored as too small; the cosines of small angles are taken as equal to unity, their sines are equal to the angles themselves; the trend of oscillations of the instantaneous centre of turn of the wide span tractor's linkage unit during its two-dimensional motion on the horizontal plane is taken into account in the calculation of the resultant moment of the forces applied by the agricultural implements, but it has no significant effect on the change of the distance to the line that passes through the points of attachment of the lower draught links, hence, that distance is assumed constant.

The set of external forces acting on the wide span tractor during its two-dimensional motion (see Fig. 1) comprises:

- rolling resistance forces on the front $\left(P_{f l 1}, P_{f r 1}\right)$ and rear $\left(P_{f l 2}, P_{f r 2}\right)$ wheels;

- tangential traction forces $P_{k l 1}, P_{k r 1}, P_{k l 2}, P_{k r 2}$ on the front and rear wheels;

- lateral forces $P_{\delta l 1}, P_{\delta l 2}, P_{\delta r 1}, P_{\delta r 2}$, which cause the drift of the front and rear wheels at angles of $\delta_{l 1}, \delta_{r 1}$ and $\delta_{l 2}, \delta_{r 2}$, respectively;

- the resultant vector $R$ (components $R_{X}$ and $R_{Y}$, respectively) and resultant moment $M_{R}$ of the forces applied by the agricultural implements, their lines passing through the centre of resistance (point $S_{p}$ ).

The lateral forces acting on the wide span tractor's wheels are in direct proportion to the angles of their drift, therefore:

$$
\begin{aligned}
& P_{\delta l 1}=k_{l 1} \delta_{l 1}, \quad P_{\delta r 1}=k_{r 1} \delta_{r 1}, \\
& P_{\delta l 2}=k_{l 2} \delta_{l 2}, \quad P_{\delta r 2}=k_{r 2} \delta_{r 2},
\end{aligned}
$$

where $k_{l 1}, k_{r 1}$ and $k_{l 2}, k_{r 2}$ - coefficients of resistance to drift of the wide span tractor's front and rear wheels, respectively.

The tractor's kinetic energy with reference to the plane XOY (Fig. 1) consists of two components representing its translation and gyration:

$$
T=\frac{\left[\left(M_{T}+M_{P}\right) V_{S}^{2}+J_{S 0} \cdot \omega_{T}^{2}\right]}{2}
$$

where $M_{T}, M_{P}$ - masses of the tractor and its process unit, respectively;

$V_{S}$ - linear velocity of the tractor relative to the plane $X O Y$;

$J_{S 0}-$ moment of inertia of the tractor and its process unit with reference to the point $S_{0}$;

$\omega_{T}-$ angular velocity of the tractor and its process unit.

It is obvious that: 


$$
V_{S}=\dot{X}_{S}, \quad \omega_{T}=\dot{\varphi}
$$

After substituting the expressions (3) and (4) into (1) and differentiating it with respect to the assumed generalized coordinates, the following system of differential equations is obtained:

$$
\left.\begin{array}{c}
\left(M_{T}+M_{P}\right) \ddot{X}_{S}=Q_{X} \\
J_{S 0} \cdot \ddot{\varphi}=Q_{\varphi}
\end{array}\right\}
$$

where $Q_{X}, Q_{\varphi}$ - generalized forces that determine the tractor's displacement on the respective generalized coordinates.

In view of the latter's smallness, the equations that determine the mentioned forces appear as follows:

$$
\begin{aligned}
& Q_{X}=\left(P_{k l 1}-P_{f l 1}\right)\left(\alpha_{l 1}-\delta_{l 1}\right)+\left(P_{k r 1}-P_{f r 1}\right)\left(\alpha_{r 1}-\delta_{r 1}\right)-\left(P_{k l 2}-P_{f l 2}\right) \delta_{l 2}- \\
& \quad-\left(P_{k r 2}-P_{f r 2}\right) \delta_{r 2}+P_{\delta l 1}+P_{\delta l 2}+P_{\delta r 1}+P_{\delta r 2}+R_{X}, \\
& Q_{\varphi}=\left(P_{\delta l 1}+P_{\delta r 1}\right)\left(L-l_{t}+D-d\right)+\left[\left(P_{k l 1}-P_{f l 1}\right)\left(\alpha_{l 1}-\delta_{l 1}\right)+\left(P_{k r 1}-P_{f r 1}\right)\left(\alpha_{r 1}-\delta_{r 1}\right)\right] \times \\
& \times\left(L-l_{t}+D-d\right)+\left(P_{k l 1}-P_{f l 1}\right) b-\left(P_{k r 1}-P_{f r 1}\right)(K-b)+P_{\delta l 1} \delta_{l 1} b-P_{\delta r 1} \delta_{r 1}(K-b)- \\
& -\left(P_{\delta l 2}+P_{\delta r 2}\right)\left(l_{t}-D+d\right)+P_{\delta l 2} \delta_{l 2} b-P_{\delta r 2} \delta_{r 2}(K-b)+\left[\left(P_{k l 2}-P_{f l 2}\right) \delta_{l 2}+\right. \\
& \left.+\left(P_{k r 2}-P_{f r 2}\right) \delta_{r 2}\right]\left(l_{t}-D+d\right)+\left(P_{k l 2}-P_{f l 2}\right) b-\left(P_{k r 2}-P_{f r 2}\right)(K-b)+ \\
& +M_{R}-R_{X}(a+d),
\end{aligned}
$$

where $L, l_{t}, K, a, b, d, D$ - design parameters of the wide span tractor, the nature of which can be understood from Fig. 1; $\alpha_{l 1}, \alpha_{r 1}-$ angular displacements of the tractor's steering wheels.

Using the method [4] of determining the drift angles of the tractor's tyres, the following is obtained:

$$
\begin{array}{ll}
\delta_{l 1}=\frac{\dot{X}_{S}+\left(L-l_{t}+D-d\right) \dot{\varphi}}{V_{0}}-\varphi-\alpha_{l 1}, & \delta_{r 1}=\frac{\dot{X}_{S}+\left(L-l_{t}+D-d\right) \dot{\varphi}}{V_{0}}-\varphi-\alpha_{r 1}, \\
\delta_{l 2}=\frac{\dot{X}_{S}-\left(l_{t}-D+d\right) \dot{\varphi}}{V_{0}}-\varphi, & \delta_{r 2}=\frac{\dot{X}_{S}-\left(l_{t}-D+d\right) \dot{\varphi}}{V_{0}}-\varphi .
\end{array}
$$

Because of the smallness of the angular displacements of the wide span tractor's steering wheels, it is assumed that $\alpha_{l 1}=\alpha_{r 1}=\alpha$. Taking that into account and substituting the expressions (8) for determining the tyre drift angles, (2) and (6) and (7) for the lateral and generalized forces into the system (5), the following differential equations of motion of the wide span tractor and its process unit on the horizontal plane are obtained:

$$
\left.\begin{array}{l}
A_{11} \ddot{X}_{S}+A_{12} \dot{X}_{S}+A_{13} \dot{\varphi}+A_{14} \varphi=f_{11} \alpha+f_{12}, \\
A_{21} \ddot{\varphi}+A_{22} \dot{\varphi}+A_{23} \varphi+A_{24} \dot{X}_{S}=f_{21} \alpha+f_{22}
\end{array}\right\}
$$

where $A_{11}=M_{T}+M_{R}$,

$$
\begin{aligned}
& A_{12}=\frac{k_{l 1}+k_{l 2}+k_{r 1}+k_{r 2}-P_{k l 1}+P_{f 11}-P_{k r 1}+P_{f r 1}-P_{k l 2}+P_{f l 2}-P_{k r 2}+P_{f r 2}}{V_{0}}, \\
& A_{13}=\frac{\left[\left(k_{l 1}+k_{r 1}-P_{k l 1}+P_{f 11}-P_{k r 1}+P_{f r 1}\right)\left(L-l_{t}+D-d\right)+\right.}{V_{0}} \times \\
& \times \frac{\left.+\left(P_{k l 2}-P_{f l 2}+P_{k r 2}-P_{f r 2}-k_{l 2}-k_{r 2}\right)\left(l_{t}-D+d\right)\right]}{}, \\
& A_{14}=-A_{12} V_{0}, \\
& A_{21}=J_{S 0},
\end{aligned}
$$


$A_{22}=\frac{\left[\left(k_{l 1}+k_{r 1}-P_{k l 1}+P_{f l 1}-P_{k r 1}+P_{f r 1}\right)\left(L-l_{t}+D-d\right)^{2}+\right.}{V_{0}} \times$

$\times \frac{\left.+\left(P_{k l 2}-P_{f l 2}+P_{k r 2}-P_{f r 2}-k_{l 2}-k_{r 2}\right)\left(l_{t}-D+d\right)^{2}\right]}{}$,

$A_{23}=-A_{13} V_{0}, \quad A_{24}=A_{13}$,

$f_{11}=k_{l 1}+k_{r 1}, \quad f_{12}=R_{X}$,

$f_{21}=\left(k_{l 1}+k_{r 1}+P_{k l 1}-P_{f l 1}+P_{k r 1}-P_{f r 1}\right) \cdot\left(L-l_{t}+D-d\right)$,

$f_{22}=M_{R}-R_{X}(a+d)+\left(P_{k l 1}-P_{f l 1}+P_{k l 2}-P_{f l 2}\right) b-\left(P_{k r 1}-P_{f r 1}+P_{k r 2}-P_{f r 2}\right) \cdot(K-b)$.

The values of the force $R_{X}$ and the moment $M_{R}$ that are parts of the system of equations (9) depends on the angle $\beta$ of the turn of the wide span tractor linkage unit's lower draught links (Fig. 2).

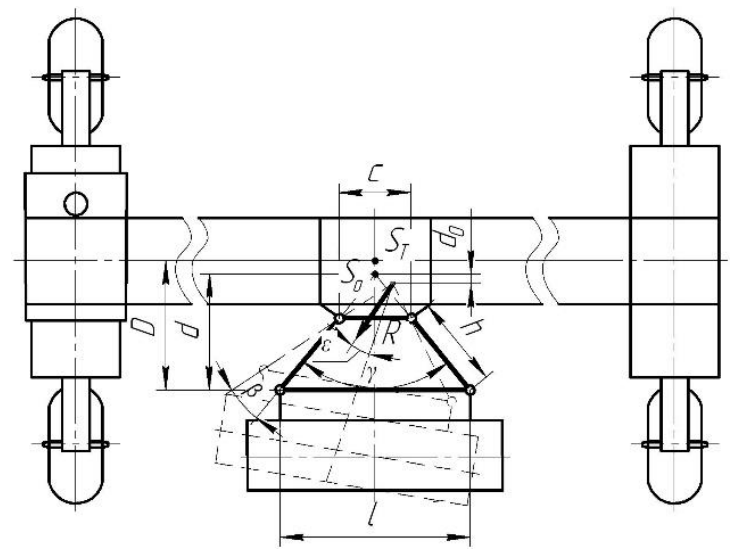

Fig. 2. Schematic model of turn of wide span tractor linkage unit's lower draught links through angle of $\beta$.

In view of the smallness of the mentioned angles shown in Fig. 2, we have:

$$
\begin{aligned}
& R_{X}=R\left[\varepsilon \pm\left(1-\frac{c}{l}\right) \beta\right] \\
& M_{R}=R\left(d_{0}+D-d\right)\left[\varepsilon \pm\left(1-\frac{c}{l}\right) \beta\right]
\end{aligned}
$$

where $\varepsilon$ - angle between the line of the tractor's traction force and the longitudinal symmetry axis of its process unit; $\beta$ - angular displacement of the wide span tractor linkage unit's lower draught links;

$d_{0}$ - longitudinal distance from the hook load application point to the instantaneous centre of turn of the tractor's linkage unit (Fig. 2);

$c, l$ - design parameters of the wide span tractor's linkage unit (Fig. 2).

In the dependences (10) and (11), the sign for the " \pm " component is determined by the accordance of the angles $\varepsilon$ and $\beta$. That is to say the sign "-" is applied, when the mentioned angles of the agricultural implement vary in opposite phase during the operation of the wide span tractor. In case of their in-phase variation the sign "+" is applied.

Thereupon, taking into account the dependences (10) and (11), the mathematical model of motion of the wide span tractor on the horizontal plane will appear as follows:

$$
\left.\begin{array}{l}
A_{11} \ddot{X}_{S}+A_{12} \dot{X}_{S}+A_{13} \dot{\varphi}+A_{14} \varphi=f_{11} \alpha+f_{12} \beta+f_{13}, \\
A_{21} \ddot{\varphi}+A_{22} \dot{\varphi}+A_{23} \varphi+A_{24} \dot{X}_{S}=f_{21} \alpha+f_{21} \beta+f_{23},
\end{array}\right\}
$$

where $A_{11}=M_{T}+M_{R}$,

$$
A_{12}=\frac{k_{l 1}+k_{l 2}+k_{r 1}+k_{r 2}-P_{k l 1}+P_{f l 1}-P_{k r 1}+P_{f r 1}-P_{k l 2}+P_{f l 2}-P_{k r 2}+P_{f r 2}}{V_{0}},
$$




$$
\begin{aligned}
& A_{13}=\frac{\left(k_{l 1}+k_{r 1}-P_{k l 1}+P_{f l 1}-P_{k r 1}+P_{f r 1}\right)\left(L-l_{t}+D-d\right)+}{V} \times \\
& \times \frac{+\left(P_{k l 2}-P_{f l 2}+P_{k r 2}-P_{f r 2}-k_{l 2}-k_{r 2}\right)\left(l_{t}-D+d\right)}{A_{14}=-A_{12} V_{0},} \\
& A_{21}=J_{s o}, \\
& A_{22}=\frac{\left(k_{l 1}+k_{r 1}-P_{k l 1}+P_{f l 1}-P_{k r 1}+P_{f r 1}\right)\left(L-l_{t}+D-d\right)^{2}+}{V_{0}} \times \\
& \times \frac{\left(P_{k l 2}-P_{f l 2}+P_{k r 2}-P_{f r 2}-k_{l 2}-k_{r 2}\right)\left(l_{t}-D+d\right)^{2}}{A_{23}}, \\
& f_{11}=-A_{13} V_{0}, \quad A_{24}=A_{13}, \quad f_{12}=R\left(1-\frac{c}{l}\right), \quad f_{13}=R \varepsilon, \\
& f_{21}=\left(k_{l 1}+k_{r 1}+P_{k l 1}-P_{f l 1}+P_{k r 1}-P_{f r 1}\right)\left(L-l_{t}+D-d\right), \\
& f_{22}=R(1-c / l)\left(d_{0}+D-a-2 d\right)+\left(P_{k l 1}-P_{f l 1}+P_{k l 2}-P_{f l 2}\right) b-\left(P_{k r 1}-P_{f r 1}+P_{k r 2}-P_{f r 2}\right)(K-b), \\
& f_{23}=R\left(d_{0}+D-a-2 d\right) \varepsilon .
\end{aligned}
$$

After applying the transformation $\frac{d}{d t}=s$, the mathematical model of the wide span tractor's two-dimensional motion in the operator format is obtained:

$$
\left.\begin{array}{l}
K_{11} X_{S}(s)+K_{12} \varphi(s)=F_{11} \alpha(s)+F_{12} \beta(s)+F_{13}(s), \\
K_{21} X_{S}(s)+K_{22} \varphi(s)=F_{21} \alpha(s)+F_{22} \beta(s)+F_{23}(s),
\end{array}\right\}
$$

where $K_{11}=A_{11} s^{2}+A_{12} s, \quad K_{12}=A_{13} s+A_{14}$,

$$
K_{21}=A_{24} s,
$$$$
K_{22}=A_{21} s^{2}+A_{22} s+A_{23} \text {, }
$$

$F_{11}=f_{11}$,

$F_{12}=f_{12}$,

$$
\begin{aligned}
& F_{13}=f_{13}, \\
& F_{23}=f_{23},
\end{aligned}
$$

$F_{21}=f_{21}$,

$$
F_{22}=f_{22} \text {, }
$$

$\frac{d}{d t}=s-$ operator of differentiation.

In the mathematical model (13), angle $\alpha$ of turn of the tractor's steering wheels (controlling action) and the angle $\beta$ of turn of the linkage unit with the process unit on the horizontal plane (perturbing action) are the input values.

The response of the dynamic system to the controlling and perturbing actions is determined by the design and process parameters of the wide span tractor, which are represented by the coefficients in the right-hand side of the system (13). In the case under consideration, we are interested in the response of the analysed dynamic system to the controlling and perturbing actions under different correlations between the $d$ and $D$ parameters. The said parameters determine the distance from the line that passes through the points of attachment of the linkage unit's lower draught links to the instantaneous centre of turn (point $S_{0}$ ) and centre of mass (point $S_{T}$ ) of the tractor. Therefore, in the process of mathematical modelling, the following three cases of the mentioned correlation between parameters are under consideration: $d<D ; d=D$ and $d>D$.

The output values of the mathematical model (13) are the transverse displacements $X_{S}$ of the wide span tractor and its heading angle $\varphi$.

The controllability of the wide span tractor's motion can be estimated with the aid of the respective amplitude frequency and phase frequency response characteristics. They are analysed, as is known, with the use of an appropriate transfer function. The function $W^{\alpha}(s)$ that characterizes the change of the tractor's heading angle $\varphi$ as a consequence of the change of its wheels' turn angle $\alpha$ can be taken in the capacity of such a function. According to the system of equations (13), it can be set out in the following form:

$$
W^{\alpha}(s)=\frac{C_{1 \alpha} s+C_{0 \alpha}}{s\left(B_{2} s^{2}+B_{1} s+B_{0}\right)},
$$


where $C_{1 \alpha}=A_{11} f_{21}, \quad C_{0 \alpha}=A_{12} f_{21}-A_{24} f_{11}$,

$B_{2}=A_{11} A_{21}, \quad B_{1}=A_{12} A_{21}+A_{11} A_{22}$,

$B_{0}=A_{12} A_{22}+A_{11} A_{23}-A_{13} A_{24}$.

The dynamic system under consideration is a follow-up system. When it responds to a controlling action, it is required that the amplitude frequency response in the operating range of frequencies is equal to unity, the phase frequency response - to zero [4].

\section{Discussion}

The analysis of the mathematical modelling data (Fig. 3 and Fig. 4) shows that the controllability of the wide span tractor design under consideration at a velocity of $2 \mathrm{~m} \cdot \mathrm{s}^{-1}$ depends little on the variation of the distance from the line that passes through the points of attachment of the linkage unit's lower draught links to the instantaneous centre of turn (point $S_{0}$ ) relative to the centre of mass (point $S_{T}$ )

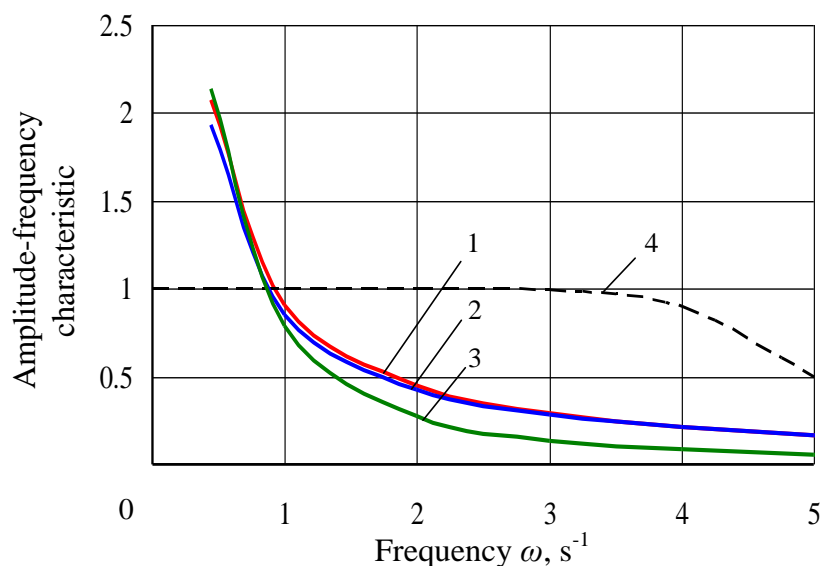

Fig. 3. Amplitude frequency response of wide span tractor to controlling action under different correlations between $d$ and $D$ parameters: 1) $d<D$; 2) $d=D$; 3) $d>D$; 4) desirable response characteristic.

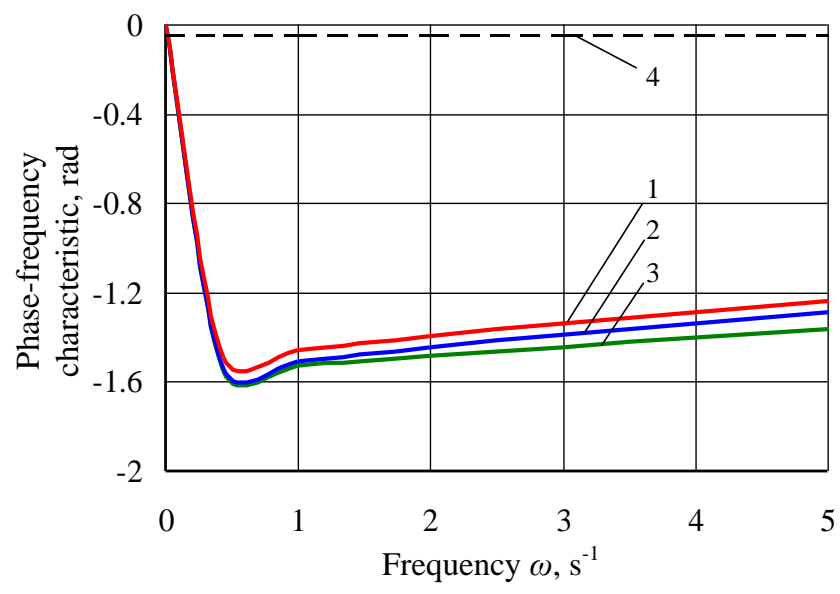

Fig. 4. Phase frequency response of wide span tractor to controlling action under different correlations between $d$ and $D$ parameters: 1) $d<D$; 2) $d=D$; 3) $d>D$;4) desirable response characteristic.

At the lower frequencies (below $0.8-0.9 \mathrm{~s}^{-1}$ ) of oscillation of the angle of turn $\alpha$ of the tractor's front wheels under possible correlations between $d$ and $D$ parameters, considerable overshoot is observed, at the greater frequencies - undershoot in the response of the dynamic system to the controlling action. In this case, the desirable frequency of oscillation of the angle of turn $\alpha$ of the tractor's front wheels lies within a range of $0.8-0.9 \mathrm{~s}^{-1}$, where the control input gain value is close to unity.

The worst characteristics of the amplitude and phase frequency response of the dynamic system to the controlling action are observed, when $d>D$. It is due to the fact that, within the operating range, the mentioned characteristics are the most distant from the desirable ones. 
The most appropriate characteristics of the amplitude and phase frequency response of the dynamic system to the controlling action are observed in case of $d<D$. In that event, the characteristics in question are the closest to the desirable ones within the operating range.

Overall, the following trend takes shape: in order to ensure the satisfactory controllability of the wide span tractor progressing at a rate of $2 \mathrm{~m} \cdot \mathrm{s}^{-1}$, the frequency of oscillation of the angle of turn of its wheels has to be within $0.8-0.9 \mathrm{~s}^{-1}$. Otherwise, the characteristics of the amplitude and phase frequency response of the dynamic system to the controlling action are close to the desirable ones, only when the instantaneous centre of turn of the wide span tractor's linkage unit is close to its centre of mass.

As may be inferred from the analysis of the phase frequency response characteristics (Fig. 4), the less is the distance $d$, the less is the lag of the wide span tractor's response to the controlling action. On the other hand, the difference between the obtained phase frequency response characteristics is insignificant and becomes notable at all only at controlling action frequencies above $0.8-0.9 \mathrm{~s}^{-1}$.

The stability of the wide span tractor's motion will be estimated in the form of its ability to resist the perturbing action represented by the linkage unit's angular displacement $\beta$ on the horizontal plane.

In accordance with the system of equations (13), the transfer function $W^{\beta}(s)$ that represents the measure of variation of the tractor's heading angle as a function of $\beta$ can be set out in the following form:

$$
W^{\beta}(s)=\frac{C_{1 \beta} \cdot s+C_{0 \beta}}{s\left(B_{2} \cdot s^{2}+B_{1} \cdot s+B_{0}\right)},
$$

where $C_{1 \beta}=A_{11} f_{22}, C_{0 \beta}=A_{12} f_{22}-A_{24} f_{12}$.

When the dynamic system under consideration responds to the perturbing action, it is required that the amplitude frequency response in the operating range of frequencies is equal to zero, the phase frequency response - goes to infinity.

As the results of the analysis show, with the increase of the distance from the line that passes through the points of attachment of the linkage unit's lower draught links to the wide span tractor's instantaneous centre of turn (parameter $d$ ) its amplitude frequency response to the perturbations in the form of the process unit's oscillation on the horizontal plane undesirably increases (Fig. 5). Although, this process becomes more or less significant only at small frequencies of oscillation of the angle $\beta\left(0.5-1.3 \mathrm{~s}^{-1}\right)$.

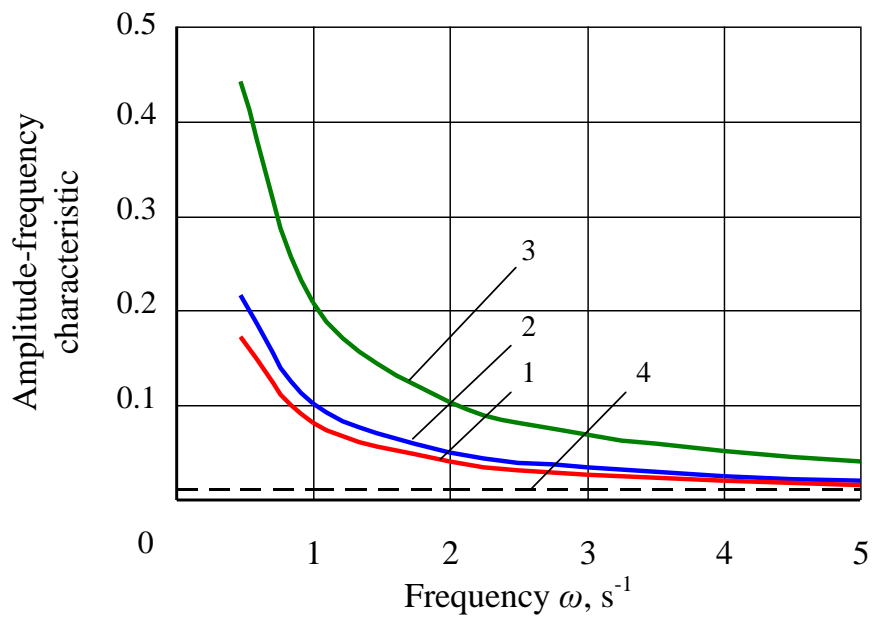

Fig. 5. Amplitude frequency response of wide span tractor to oscillation of angular displacement of its process unit under different correlations between $d$ and $D$ parameters: 1) $d\langle D$;2) $d=D$; 3) $d\rangle D$; 4) desirable response characteristic.

It should be noted also that the wide span tractor's response to the perturbing action in this case undesirably, even though less intensively, decreases (Fig. 6).

From the point of view of the dynamic system's response to the perturbing action, the most desirable case of the correlation between the parameters under discussion is when $d>D$. The latter condition indicates that the instantaneous centre of turn of the wide span tractor's linkage unit has to be close to its centre of mass.

The mentioned requirement can be met if such an angle $\gamma$ of convergence of the wide span tractor linkage unit's lower draught links is chosen that the instantaneous centre of turn of the linkage unit is situated close to the tractor's centre of mass. In practice, that can be achieved by means of setting the appropriate value of $c$ (Fig. 2). In case of the equality $d=D$, the parameter $c$ in Fig. 2 has to be as follows: 


$$
c=l\left(1-\frac{2 h}{\sqrt{l^{2}+4 D^{2}}}\right) .
$$

At the same time, the convergence angle $\gamma$ between the lower draught links of the wide span tractor's linkage unit has to be equal to:

$$
\gamma=2 \tan ^{-1}\left(\frac{1}{2 D}\right)
$$

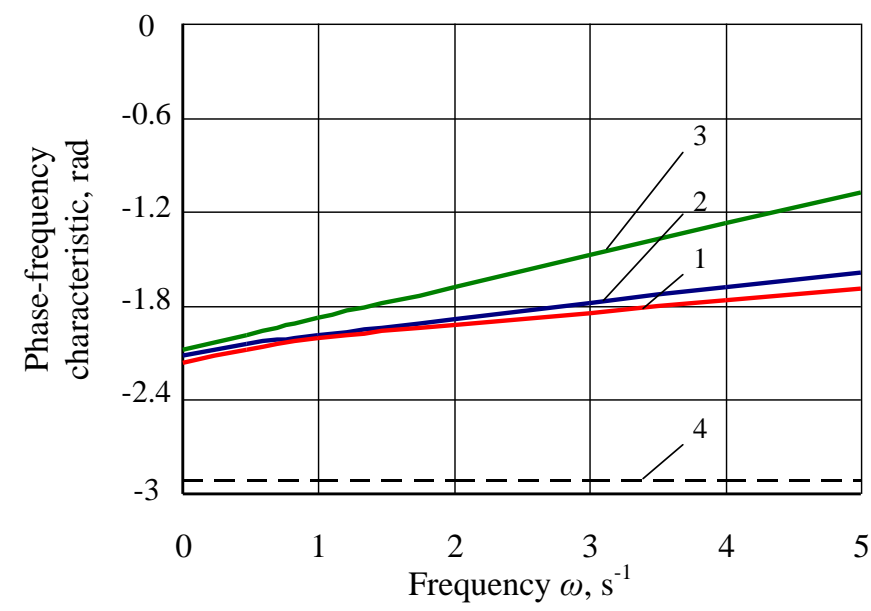

Fig. 6. Phase frequency response of wide span tractor to oscillation of angular displacement of its process unit under different correlations between $d$ and $D$ parameters: 1) $d\langle D$; 2) $d=D$; 3) $d\rangle D$; 4) desirable response characteristic.

The analysis of the dependences (14) and (15) for the linkage units in the maximum categories $4 \mathrm{~N}$ and 4 according to ISO 730:2009 has shown that, as the distance $D$ from the line that passes through the points of attachment of the lower draught links to its centre of mass increases, the required increase of the length $c$ changes disproportionately (Fig. 7). The same disproportion is observed in this case in the decrease of the lower draught links' convergence angle $\gamma$. Overall, as the length $D$ increases from 0.6 to $2.6 \mathrm{~m}$, the distance $c$ grows from $0.02 \mathrm{~m}$, which in practice corresponds to the complete bringing of the ends of the wide span tractor linkage unit's lower draught links to one point, to $0.77 \mathrm{~m}$. At the same time, the angle $\gamma$ decreases from 85 to $24 \mathrm{deg}$.

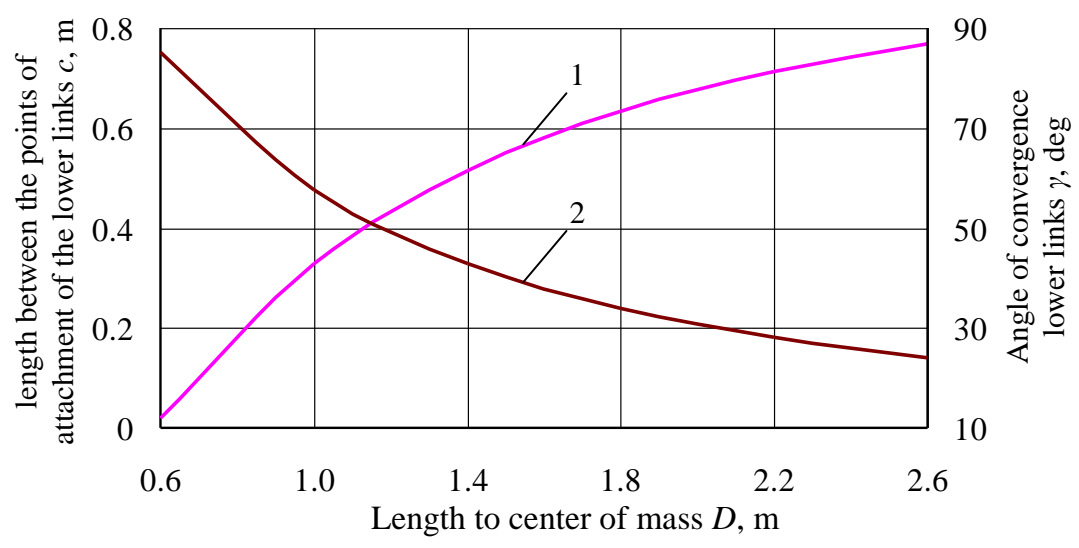

Fig. 7. Dependence relations between distance between points of attachment of wide span tractor linkage unit's lower draught links (1) or angle of convergence of lower draught links (2) and distance to wide span tractor's centre of mass

The undertaken investigation has shown that, in order to provide for the sufficient angular mobility of the wide span tractor's process unit with respect to its frame, considerable attention must be paid to the correlation between the distance between the points of attachment of the linkage unit's lower draught links and the distance to its centre of mass. Moreover, the closeness of the linkage unit's position to the wide span tractor's centre of mass implies that the required distance between the points of attachment of linkage unit's lower draught links is so small that it is equivalent to their merging to one point. Hence, the design of a multi-purpose linkage unit for wide span tractors has to provide for setting it up with the use of both the three-point and two-point configurations. 


\section{Conclusions}

1. Wide span tractors (vehicles) are lately gaining interest in the world, which allows implementing a controlled traffic farming technology. The problem is to ensure stable motion of the wide span tractor (vehicle). The design of its linkage has to provide for its independent turning on the horizontal plane. This study investigates into the details of hitching the wide span tractors with agricultural machines and implements.

2. Concerning the response of the wide span tractor to the controlling action (angular displacement of the steering wheels) and the perturbing action (angular displacement of the linkage unit on the horizontal plane), the completed investigation has shown that the most desirable set-up of the tractor's linkage unit is when the instantaneous centre of turn situated at the point of convergence of the centrelines of the lower draught links is close to the tractor's centre of mass.

3. In order to provide for the sufficient angular mobility of the wide span tractor's process unit with respect to the tractor's frame, considerable attention must be paid to the correlation between the distance between the points of attachment and the angle of convergence of the linkage unit's lower draught links, on the one hand, and the distance to its centre of mass, on the other hand. As the latter decreases, the required distance between the points of attachment of the linkage unit's lower draught links can become so small that that will be equivalent to their merging to one point. In this case, the angle of convergence of the lower draught links will reach a value of 85 degrees.

4. The accomplished research into the linkage units of the maximum categories $4 \mathrm{~N}$ and 4 in accordance with ISO 730:2009 has shown that the design of a multi-purpose linkage unit for wide span tractors has to provide for setting it up with the use of both the three-point and two-point configurations.

5. Future research plans are aimed at: a) optimisation of the vertical load of the wheels of the wide span tractor; b) improvement in the pulling characteristics of the wide span tractor, and c) decrease in the influence of soil treading.

\section{References}

[1] Bindi, I.; Blackwell, P., Riethmuller, G.; Davies, S.; Whitlock, A. \& Neale, T. (2013). This Controlled Traffic Farming Technical Manual. It updates the Tramline Farming Systems: Technical Manual Bulletin 4607. Published by the Department of Agriculture and food, Western Australia. 78 p.

[2] Blackwell, P.; McKenzie, D; Webb, B.; Lemon, J.; Barber, P.; Fretwell, G.; Bignell, G. \& Moffat, N. (2004). Compaction of heavy soils by cropping traffic and estimated benefits of tramline farming. Agribusiness Crop Updates, Perth.

[3] Bochtis, D.D.; Sørensen, C.G.; Busatob, P.; Hameed, I.A.; Rodias, E.; Green, O. \& Papadakis, G. (2010). Tramline establishment in controlled traffic farming based on operational machinery cost. Biosystems Engineering, Vol. 107, No. 3, pp. 221-231, ISSN 1537-5110, DOI: 10.1016/j.biosystemseng.2010.08.004.

[4] Bulgakov, V.; Adamchuk, V.; Kuvachov, V.; Arak, M. \& Olt, J. (2017). Study into movement of wide span tractors (vehicles) used in controlled traffic farming, Proceedings of the 28th DAAAM International Symposium, pp.01990208, B. Katalinic (Ed.), Published by DAAAM International, ISBN 978-3-902734-11-2, ISSN 1726-9679, Vienna, Austria, DOI: 10.2507/28th.daaam.proceedings.027.

[5] Chamen T. (2015). Controlled traffic farming - from world wide research to adoption in Europe and its future prospects. Acta Technologica Agriculturae Nitra 3, pp. 64-73.

[6] Evans, T.A.; Dawes, T.Z.; Ward, P.R. \& Lo, N. (2011). Ants and termites increase crop yield in a dry climate. Nature Communications, 2:262 doi: 10.1038/ncomms1257.

[7] Onal I. (2012). Controlled Traffic farming and Wide Span Tractors. Journal of Agricultural Machinery Science Vol. 8, No 4, pp. 353-364.

[8] Pedersen, H.H. (2011). Harvest Capacity Model for a Wide Span Onion Bunker Harvester. Automation and System Technology in Plant Production, CIGR section V \& NJF section VII conference, pp. 27-36.

[9] Pedersen, H.H., Oudshoorn, F.W., McPhee, J.E., Chamne, W.C.T. (2016) Wide span - Re-mechanising vegetable production. Acta Horticulturae, Vol. 1130, pp. 551-557, ISSN: 05677572, DOI: 10.17660/ActaHortic.2016.1130.83.

[10] Popp, K., Schiehler, W. (2010). Ground Vehicle Dynamics. Springer. ISBN 978-3-540-68553-1.

[11] Rajamani, R. (2012). Vehicle dynamics and control. Springer. ISBN 978-1-4614-1433-9.

[12] Pullen D.W.M., Cowell P.A. (2000) Prediction and experimental verification of the hoe path of a rear-mounted interrow weeder. Journal of Agricultural Engineering Research. Vol. 77, Issue 2, pp. 137-153. ISSN: 00218634, DOI: $10.1006 /$ jaer.1998.0346.

[13] Tullberg J.N. (2000), Wheel traffic effects on tillage draft. Journal of Agricultural Engineering Research, Vol. 75, No. 4, pp. 375-382, ISSN 0021-8634, DOI: 10.1006/jaer.1999.0516.

[14] Tullberg, J.N.; Yule, D.F. \& McGarry D. (2007). Controlled traffic farming - from research to adoption in Australia. Soil and Tillage Research, Vol. 97, No. 2, pp. 272-281, ISSN 0167-1987, DOI: 10.1016/j.still.2007.09.007.

[15] Wang, Q., Zhao H., He J., Chen, W., Cao, X., Rasaily, R.G. (2016) Design and experiment of blades-combined no and minimum-till wheat planter under controlled traffic farming system. Transactions of the Chinese Society of Agricultural Engineering. Vol. 32, Issue 17, pp. 12-17, ISSN: 10026819 , DOI: 10.11975/j.issn.10026819.2016.17.002.

[16] Webb, B., Blackwell, P., Riethmuller, G. \& Lemon, J. (2004). Tramline farming systems: technical manual, Bulletin 4607, Department of Agriculture, Western Australia.

[17] Wong, J.Y. (2008). Theory of ground vehicles. John Wiley \& Sons, ISBN 978-0-470-17038-0. 